June 24, 2018 21:0 WSPC/INSTRUCTION FILE fb-ijmpa revised

International Journal of Modern Physics A

(C) World Scientific Publishing Company

\title{
Expectation of forward-backward rapidity correlations in $p+p$ collisions at the LHC energies
}

\author{
Ajay Kumar Dash \\ Universidade Estadual de Campinas, \\ 13083-970, Campinas, Sao PauloCity, Brazil \\ ajayd@ifi.unicamp.br \\ Durga Prasad Mahapatra \\ Institute of Physics, \\ Sachivalaya Marg, Bhubaneswar - 751005, India \\ dpm@iopb.res.in \\ Bedangadas Mohanty \\ Variable Energy Cyclotron Centre \\ 1/AF, Bidhan Nagar, Kolkata - 700064, India \\ bmohanty@vecc.gov.in
}

Received Day Month Year

Revised Day Month Year

Forward-backward correlation strength $(b)$ as a function of pesudorapidity intervals for experimental data from $p+\bar{p}$ non-singly diffractive collisions are compared to PYTHIA and PHOJET model calculations. The correlations are discussed as a function of rapidity window $(\Delta \eta)$ symmetric about the central rapidity as well as rapidity window separated by a gap $\left(\eta_{\text {gap }}\right)$ between forward and backward regions. While the correlations are observed to be independent of $\Delta \eta$, it is found to decrease with increase in $\eta_{g a p}$. This reflects the role of short range correlations and justifies the use of $\eta_{g a p}$ to obtain the accurate information about the physics of interest, the long range correlations. The correlation strength from PYTHIA are in agreement with the available experimental data while those from the PHOJET give higher values. For $p+p$ collisions at $\sqrt{s}=7$, 10 and $14 \mathrm{TeV}$, the correlation strength from PHOJET are lower compared to those from PYTHIA, this is in contrast to the observations at lower energies. The experimental $b$ value shows a linear dependence on $\ln \sqrt{s}$ with the maximum value of unity being reached at $\sqrt{s}=16 \mathrm{TeV}$, beyond the top LHC energy. However calculations from the PYTHIA and PHOJET models indicate a deviation from linear dependence on $\ln \sqrt{s}$ and saturation in the $b$ values being reached beyond $\sqrt{s}=1.8 \mathrm{TeV}$. Such a saturation in correlation values could have interesting physical interpretations related to clan structures in particle production. Strong forward-backward correlations are associated with cluster production in the collisions. The average number of charged particles to which the clusters fragments, called the cluster size, are found to also increase linearly with $\ln \sqrt{s}$ for both data and the models studied. The rate of increase in cluster size vs. $\ln \sqrt{s}$ from models studied are larger compared to those from the data and higher for PHOJET compared to PYTHIA. Our study indicates that the forward-backward measurements 
will provide a clear distinguishing observable for the models studied at LHC energies.

Keywords: Forward-Backward correlations, Long range correlations, cluster formation, $\mathrm{p}+\mathrm{p}$ collisions at $\mathrm{LHC}$

PACS numbers: $1.200,1.300$

\section{Introduction}

Understanding the mechanism of particle production in $p+p$ collisions is one of the first goals of the experiments at the Large Hadron Collider (LHC). Several new results from the LHC experiments in terms of particle multiplicities at $\sqrt{s}=$ $0.9,2.36,2.76$ and $7 \mathrm{TeV}$ are being compared to various models of particle production 2]3|4|5|6|7. The most popular models for comparison to data from $p+p$ collisions being PYTHIA 8/9|10|11 and PHOJET 12|13. The PHOJET model combines the ideas based on a dual parton model (DPM) 1415 on soft process of particle production and uses lowest-order perturbative QCD for hard process. Regge phenomenology is used to parameterize the total, elastic and inelastic cross-sections. The initial and final state parton shower are generated in leading log-approximation. PYTHIA on the other hand uses string fragmentation as a process of hadronization and tends to use the perturbative parton-parton scattering for low to high $p_{T}$ particle production. Initial results have shown that both models do not have perfect agreement with multiplicity measurements at LHC energies studied so far 21314|5|617. However it may be mentioned that several of these models in turn are used to obtain various correction factors for the experimental measurements. In this paper we suggest that the correlations between the particles produced in forward and backward rapidities can be used to discriminate between the various models of particle production, more reliably at the LHC energies.

The forward-backward correlations previously observed had several physical interpretations. The correlations over small range in rapidity are believed to be dominated by short-range correlations as due to resonance decays, those occurring over large rapidity range could be interpreted to be due to multiple parton interactions. In early 1985, a statistical interpretation of the forward-backward correlations observed in ISR energies was provided 16. The interpretation was based on clusters being produced in these hadronic collisions according to a negative binomial distribution and the final hadrons are a result of the decay of these clusters. An extended version of such a statistical scenario can be found in Ref. 17. A more dynamical interpretation was provided based on the DPM as in Ref. 18 . The experimental data was also interpreted in terms of a model based on a unitarized model which included soft and semi-hard components (minijets). In such a model the average number of particles produced was proportional to the effective number of inelastic collisions. The increase of forward-backward correlations with $\sqrt{s}$ was fully generated by the superposition of the different impact parameter contributions to the inelastic cross section 19. The extension of this interesting idea is to consider a weighted superposition of two classes of events in hadronic collisions: soft and semi-hard processes. 
The behavior of the semi-hard component on observed forward-backward correlations can lead to interesting interpretation in terms of the clan structure of particle production as discussed in Refs 20 . The authors of Refs 20 envision a possibility of formation of new species of clans at LHC and a possible phase transition in clan production mechanism.

Recently the forward-backward correlations have been related to the simplest form of partonic interaction that exhibits back-to-back correlation. Such a formulation is found to give a fairly good description of data from STAR Collaboration in $p+p$ collisions at $\sqrt{s}=200 \mathrm{GeV}$ 21. Studies of forward-backward correlations provide baseline measurements to look for long range rapidity correlation in heavy-ion collisions. STAR experiment has recently made this relative (comparison between $p+p$ and different collision centrality $\mathrm{Au}+\mathrm{Au}$ collisions at the center of mass energies of $200 \mathrm{GeV}$ ) measurements to claim the existence of a large long range correlation in central $\mathrm{Au}+\mathrm{Au}$ collisions 22. Although the experimental data cannot differentiate whether the actual underlying mechanism is due to those in a dual parton model $14 \mid 15$ DPM or a Color Glass Condensate 23124 , both however require that the long range correlations are produced by multiple parton-parton interactions. Further it is argued that the clustering of color sources could lead to forward-backward correlations 25 . Hence the measurement of the long range forward backward correlations in the multiplicity of produced particles in high energy collisions will give us insight into the space-time dynamics of the collision.

In this paper, we first review the existing experimental data on forward-backward correlations, discuss the model calculations and methods used to extract these correlations. This is followed by comparison of the experimental data on forwardbackward correlations with those from PYTHIA and PHOJET models, expectations at top LHC energies and interpretation of the data in terms of cluster production in high energy hadronic collisions. We find that the forward-backward correlations in $p+p$ collisions at $\sqrt{s}=7,10,14 \mathrm{TeV}$ will help differentiate various models and hence the underlying particle production mechanism. Saturation of correlation strength at LHC energies could indicate new physics.

\section{Experimental data, Model Simulation and Correlations}

The experimental charged particle data reviewed in this paper are from the E735 and UA5 collaboration. These data are for $p+\bar{p}$ collisions and corresponds to nonsingly diffractive events. The E735 data 26 corresponds to $\sqrt{s}=300,546,1000$ and $1800 \mathrm{GeV}$ while those from UA5 27 corresponds to $\sqrt{s}=200,546$ and $900 \mathrm{GeV}$. The pesudorapidity acceptance range for the E735 experimental data is $|\eta|<3.25$ and those for UA5 is $|\eta|<4.0$.

The model results presented in this paper are from PYTHIA (Version 6.4) and PHOJET (Version 1.1, uses jetset74 from PYTHIA) with default settings. The event type selected are non-singly diffractive as for the existing measurements. For comparison to existing data the simulations are done for $p+\bar{p}$ collisions with the 


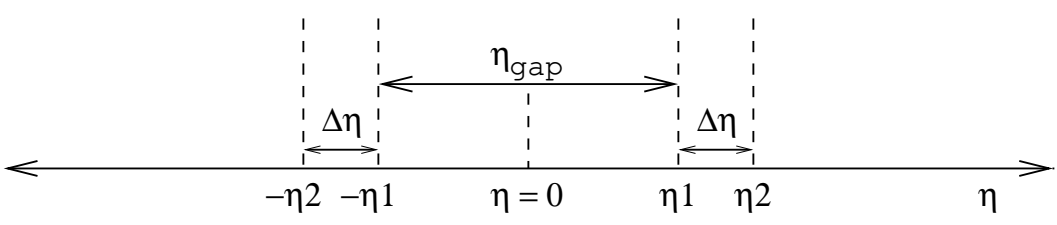

Fig. 1. Definition of $\Delta \eta$ and $\eta_{\text {gap }}$ used in forward-backward correlation studies.

experimental acceptances included. For predictions at LHC energies of $\sqrt{s}=10$ and $14 \mathrm{TeV}$ the model calculations are done for $p+p$ collisions. A realistic transverse momentum cut of above $100 \mathrm{MeV}$ for charged particles are applied.

The forward-backward correlations can be obtained by two methods.

- If $N_{b}$ is the multiplicity in backward hemisphere and $N_{f}$ is the corresponding multiplicity in forward hemisphere for the same event, then the correlation co-efficient can be obtained by plotting $\left\langle N_{b}\right\rangle\left(N_{f}\right)$ vs. $N_{f}$. Where $\langle.$. denotes event average. The resultant distribution can be fitted to a linear function as $\left\langle N_{b}\right\rangle\left(N_{f}\right)=a+b N_{f}$, to obtained the correlation strength, $b$.

- The correlation coefficient can also be defined as $b=\frac{\left\langle N_{f} N_{b}\right\rangle-\left\langle N_{f}\right\rangle\left\langle N_{b}\right\rangle}{\left\langle N_{f}^{2}\right\rangle-\left\langle N_{f}\right\rangle}$. Most of the results presented in this paper uses this method for calculating $b$. For the model results presented we have explicitly checked and found there is good agreement between the two methods.

Before we proceed towards discussion of the experimental data and results from model calculations, we discuss few relevant experimental aspects. The forwardbackward correlation results for a given $\sqrt{s}$ are usually presented as a function $\Delta \eta$ and $\eta_{\text {gap }}$. Fig. 1 shows how these quantities are defined. The $\Delta \eta$ corresponds to calculation of correlation coefficient in a symmetric window about the central rapidity. Increasing $\Delta \eta$ window includes the contributions from smaller $\Delta \eta$ window. The results as a function of $\eta_{\text {gap }}$ corresponds to correlation co-efficient being calculated in some fixed $\Delta \eta$ window separated by a gap in rapidity between forward and backward regions by an amount $\eta_{\text {gap }}$.

Most of the existing forward-backward correlation results at high energies are from $p+\bar{p}$ collisions. The current work presents the predictions of the correlation co-efficient from PYHTIA and PHOJET models in $p+p$ collisions at LHC energies and compares them to extrapolations of results from $p+\bar{p}$ collisions. So it is essential to check using models if a difference is expected in correlation values between $p+p$ and $p+\bar{p}$ collisions at a given $\sqrt{s}$. Note however that the STAR Collaboration has measured the strength of charged particle forward-backward multiplicity correlation, $b$ in $p+p$ collision at $\sqrt{s}=200 \mathrm{GeV} 28$. It is about $3-4$ times smaller than the one measured by UA5 in $p+\bar{p}$ collision at the same energy apparently 27 . The eta gap in STAR is 0.2 where as in UA5 it is 0.5 . Also both the experimental 

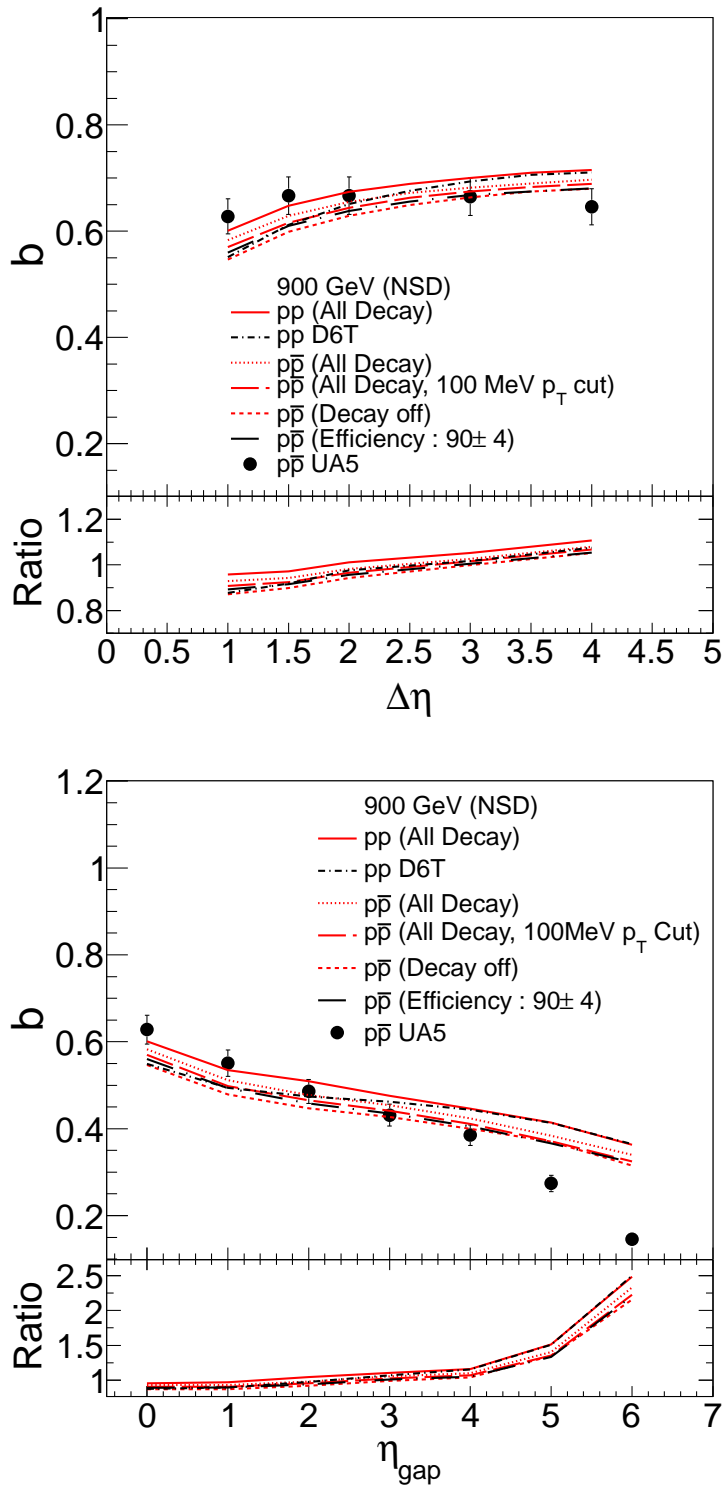

Fig. 2. Forward-backward correlation co-efficient from PYTHIA at $\sqrt{s}=900 \mathrm{GeV}$ as a function of $\Delta \eta$ and $\eta_{g a p}$. The results are from non-singly diffractive events and presented for both $p+p$ and $p+\bar{p}$ collisions. The effect of weak decay, lower transverse momentum cut off of $100 \mathrm{MeV} / c$, typical charged particle detector efficiency, different model parameter are studied. For comparison the results from $p+\bar{p}$ collisions in UA5 experiment are also shown. The bottom panels of the figure shows the ratio of the correlations from simulations with various conditions to those measured by UA5 experiment.

acceptances are different. STAR has $|\eta|<1.0$ where as UA5 has $|\eta|<4$. 
Fig. 22 shows the correlation co-efficient $(b)$ calculated using non-singly diffractive events from PYTHIA model at $\sqrt{s}=900 \mathrm{GeV}$ as a function of $\Delta \eta$ for $p+p$ and $p+\bar{p}$ collisions. No appreciable difference is observed between the two colliding systems for correlations studied as a function of $\Delta \eta$ and $\eta_{g a p}$. Also shown for comparison are the charged particle correlation co-efficient results from UA5 experiment. Usually due to experimental limitations the analysis is carried out with a lower $p_{\mathrm{T}}$ cut off. As seen in Fig. 22 a typical experimental cut-off of $100 \mathrm{MeV}$ on $p_{\mathrm{T}}$ does not seem to change the correlation values as a function of $\Delta \eta$ and $\eta_{g a p}$. We have also investigated the effect of weak decay particle contributions to this analysis. The difference between weak decay on and weak decay off for $p+\bar{p}$ collisions is noticeable for smaller $\Delta \eta$ and the difference vanishes as we go to larger $\Delta \eta$. This is along expected lines, as decay effect will introduce short-range correlations. The effect of finite charged particle detection efficiency has been studied by varying it between $86 \%$ to $94 \%$, no appericiable change is observed. The effect of a different tuned version of PYTHIA (D6T) has also been investigated. This also does not seem to affect the observed correlations by a large amount. The bottom panel of the figure shows the ratio of the correlation from simulation with various effects to the correlation measured by UA5 experiment. One observes that for the study with respect to $\Delta \eta$ the variations lie within $20 \%$. For the study with respect to the $\eta_{\text {gap }}$, the ratio is close to unity upto a value of $\eta_{\text {gap }} \quad 4$ units, then the simulation starts to diverge away from the measurements.

\section{Correlations in $\Delta \eta$ and $\eta_{\text {gap }}$}

Fig 3 shows the correlation strength $(b)$ as a function of $\Delta \eta$ for non-singly diffractive events in $p+\bar{p}$ collisions from E735 26 and UA5 27 experiments at various $\sqrt{s}$ compared to corresponding results from PYTHIA and PHOJET model calculations. The following observations can be made: (a) The correlation strength both in experimental data and simulations are observed to be almost independent of $\Delta \eta$, (b) correlations seem to increase with $\sqrt{s}$, and (c) PYTHIA model calculations agrees well with the experimental data, while PHOJET tends to give higher correlations for all the measured $\sqrt{s}$. This can be seen from the ratio of the correlation value from model to data in the bottom panel of the Fig. 3. As the larger $\Delta \eta$ includes contribution from smaller $\Delta \eta$ intervals, such a data may not provide accurate information about long range correlations. These correlations could include significant short-range correlations.

Fig 4 shows the correlation strength $(b)$ as a function of $\eta_{\text {gap }}$. The value of $b$ decreases with increase in $\eta_{\text {gap }}$ indicating the diminishing contribution from shortrange correlations. With a $\eta_{\text {gap }}$ of around 2 units still significant correlation at the level of $30-40 \%$ are observed. Both the models reproduce the decreasing trend of the correlations with increasing $\eta_{\text {gap }}$. PYTHIA model calculations has better agreement with the measurements, while PHOJET model in general over estimates the correlation strength at most of the $\sqrt{s}$, except for $\sqrt{s}=1.8 \mathrm{TeV}$. This can be 

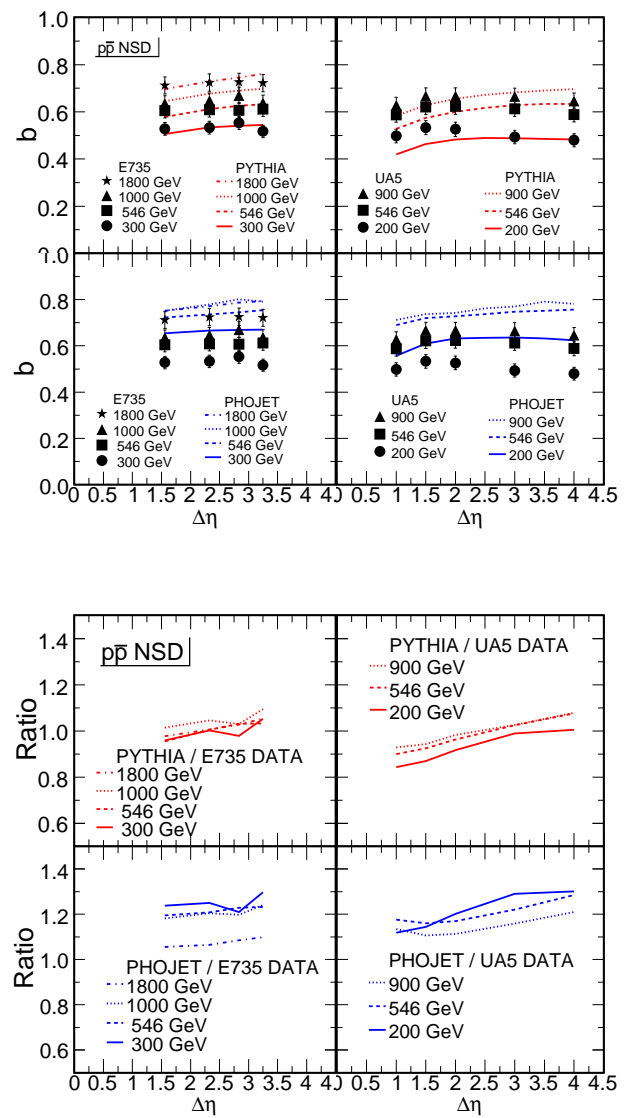

Fig. 3. Top panel: Forward-backward correlation strength (b) as a function of $\Delta \eta$ for $p+\bar{p}$ nonsingly diffractive collisions at various $\sqrt{s}$ from E735 and UA5 experiments. The measurements are compared to PYTHIA and PHOJET model calculations. Bottom panel: The ratio of model calculations to experimental data.

seen from the ratio of correlations from models to that from data as shown in the bottom panel of Fig. 4 . The agreement with the highest energy data by both the models poses the question whether at LHC energies the current measurements will have the distinguishing power. To investigate this possibility, we now discuss the predictions at three LHC energies of $\sqrt{s}=7,10$ and 14 TeV from PYTHIA and PHOJET models.

Fig. 5 and 6 shows the prediction of forward-backward correlation strength $(b)$ from PYTHIA and PHOJET models as a function of $\Delta \eta$ and $\eta_{\text {gap }}$ for $p+p$ collisions at $\sqrt{s}=7,10$ and $14 \mathrm{TeV}$. Also shown for understanding are the energy dependence trend of the existing correlation measurements from $p+\bar{p}$ collisions. In general both models predict a higher but constant value of $b$ as a function of $\Delta \eta$ at $\sqrt{s}=7,10$ 

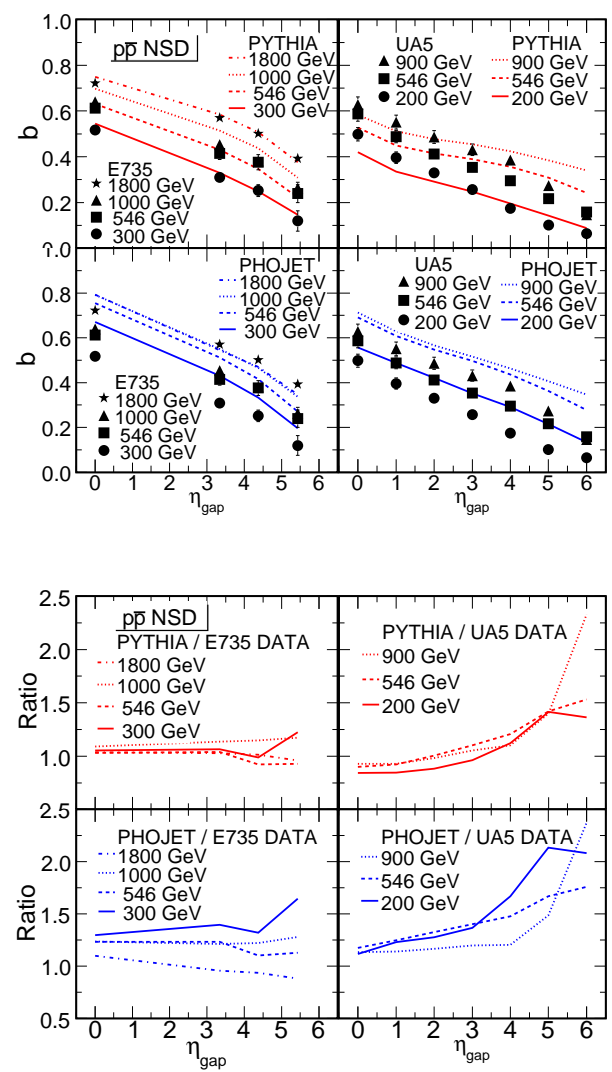

Fig. 4. Top panel: Forward-backward correlation strength $(b)$ as a function of $\eta_{\text {gap }}$ for $p+\bar{p}$ nonsingly diffractive collisions at various $\sqrt{s}$ from E735 and UA5 experiments. The measurements are compared to PYTHIA and PHOJET model calculations. Bottom panel: The ratio of model calculations to experimental data.

and $14 \mathrm{TeV} p+p$ collisions, similar to the trend seen at lower energies $p+\bar{p}$ collisions. Although PYTHIA tends to predict a slightly increasing trend at larger $\Delta \eta$. Both models also predict a decreasing trend of $b$ as a function of $\eta_{\text {gap }}$ at $\sqrt{s}=7,10$ and $14 \mathrm{TeV}$ as was observed for lower energies. The decrease in $b$ with respect to $\eta_{\text {gap }}$ from PYTHIA is slower compared to that from PHOJET at 7, 10 and $14 \mathrm{TeV}$. Both models clearly suggest that the correlation strength should saturate at higher energies, with PHOJET indicating that saturation could occur as early as $\sqrt{s}=1.8$ $\mathrm{TeV}$.

The energy dependence of the forward-backward correlation can be seen in the Fig. 7. All available experimental data from ISR 29 , UA5 27 and E735 26 Collaborations shows a linear increase in the correlation value with beam energy. The best description of the data is obtained as $\alpha+\beta \ln \sqrt{s}$, where $\alpha=-0.18 \pm 0.02$ and 

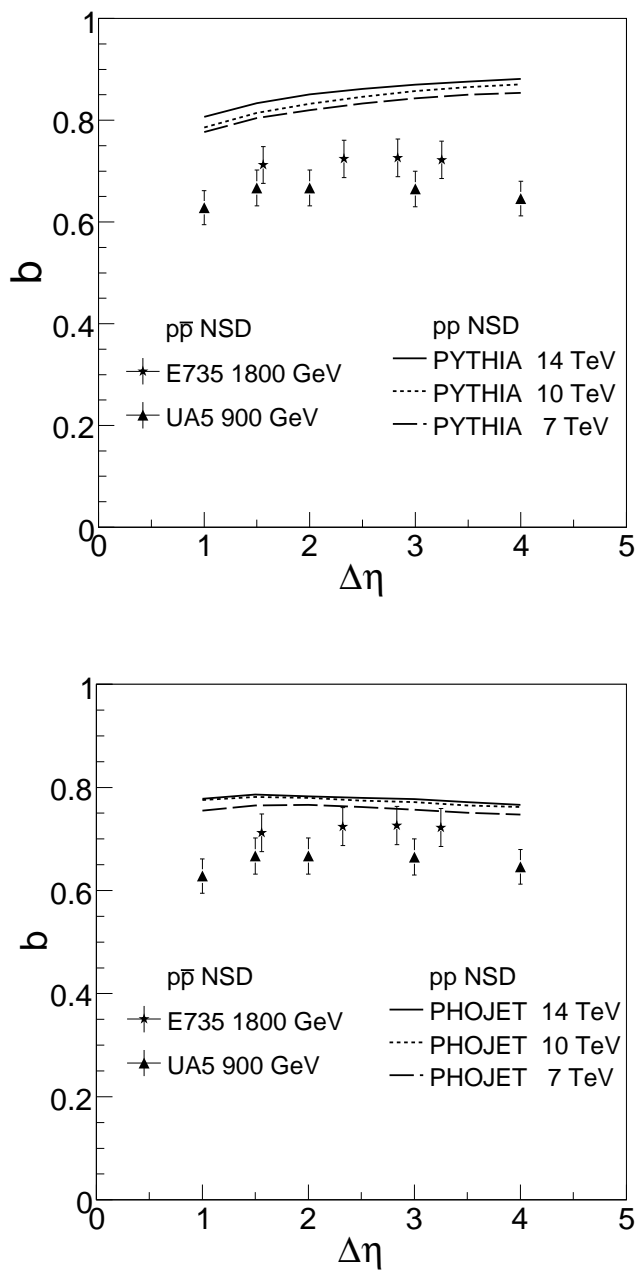

Fig. 5. Expected forward-backward correlation strength $(b)$ estimated from PYTHIA and PHOJET models as a function of $\Delta \eta$ for $p+p$ collisions at LHC energies of $\sqrt{s}=7,10$ and $14 \mathrm{TeV}$. Also shown for understanding are the energy dependence of the correlations from the existing measurements in $p+\bar{p}$ collisions.

$\beta=0.122 \pm 0.005$ are the fit parameters. For the $\sqrt{s}>200 \mathrm{GeV}$ the correlation values from PYTHIA model are in good agreement with the measurements, but those from PHOJET model are higher. However interestingly, at the LHC energies the estimates for $b$ are lower from PYTHIA compared to PHOJET. If the $\alpha$ $+\beta \ln \sqrt{s}$ dependence of $b$ on $\sqrt{s}$ holds then the maximum correlation value of 1 will be attained for $\sqrt{s} \sim 16 \mathrm{TeV}$, beyond LHC energies. Beyond which the value is expected to saturate. But the model estimates show that the values of $b$ could 

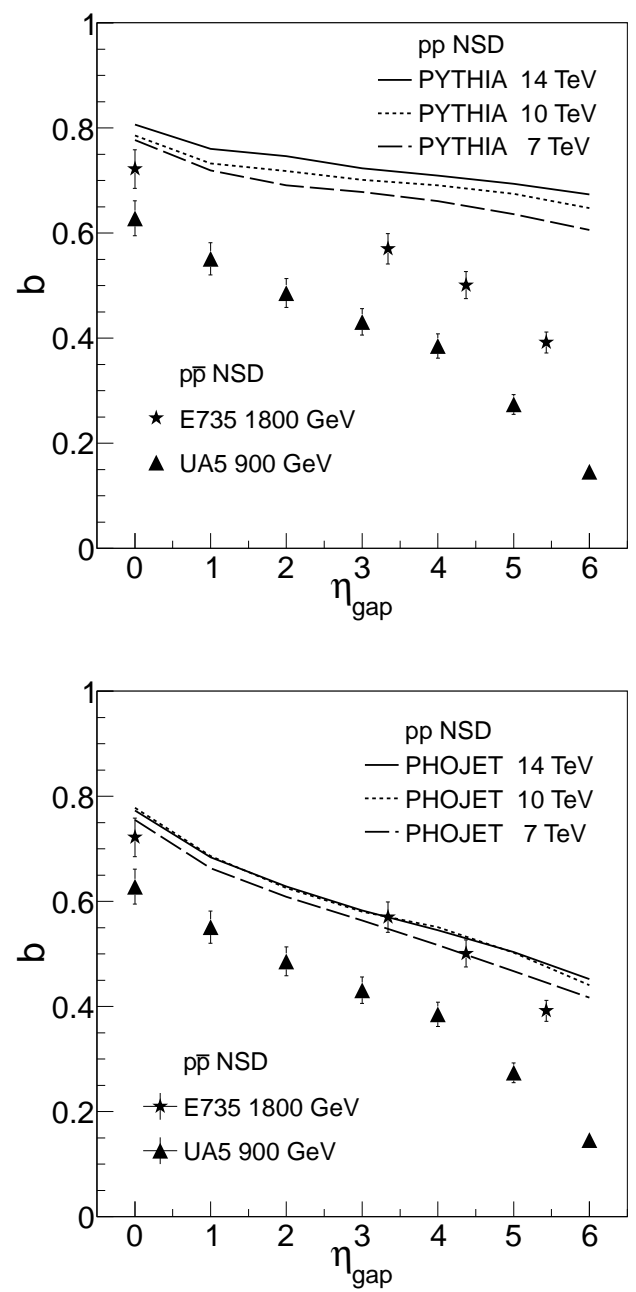

Fig. 6. Expected forward-backward correlation strength (b) estimated from PYTHIA and PHOJET models as a function of $\eta_{\text {gap }}$ for $p+p$ collisions at LHC energies of $\sqrt{s}=7,10$ and $14 \mathrm{TeV}$. Also shown for understanding are the energy dependence of the correlations for the existing measurements in $p+\bar{p}$ collisions.

saturate or drop earlier around $\sqrt{s}=1.8 \mathrm{TeV}$. The PHOJET models predicting a saturation/drop in correlation values starting at a somewhat smaller $\sqrt{s}$ compared to that predicted from PYTHIA. New data at LHC energies will provide a clear picture.

Saturation or drop in the energy dependence of the correlation values could have interesting physical consequences as is discussed in Refs 20 . In this geometric picture, the energy dependence of $b$ is understood based on the superposition of two 


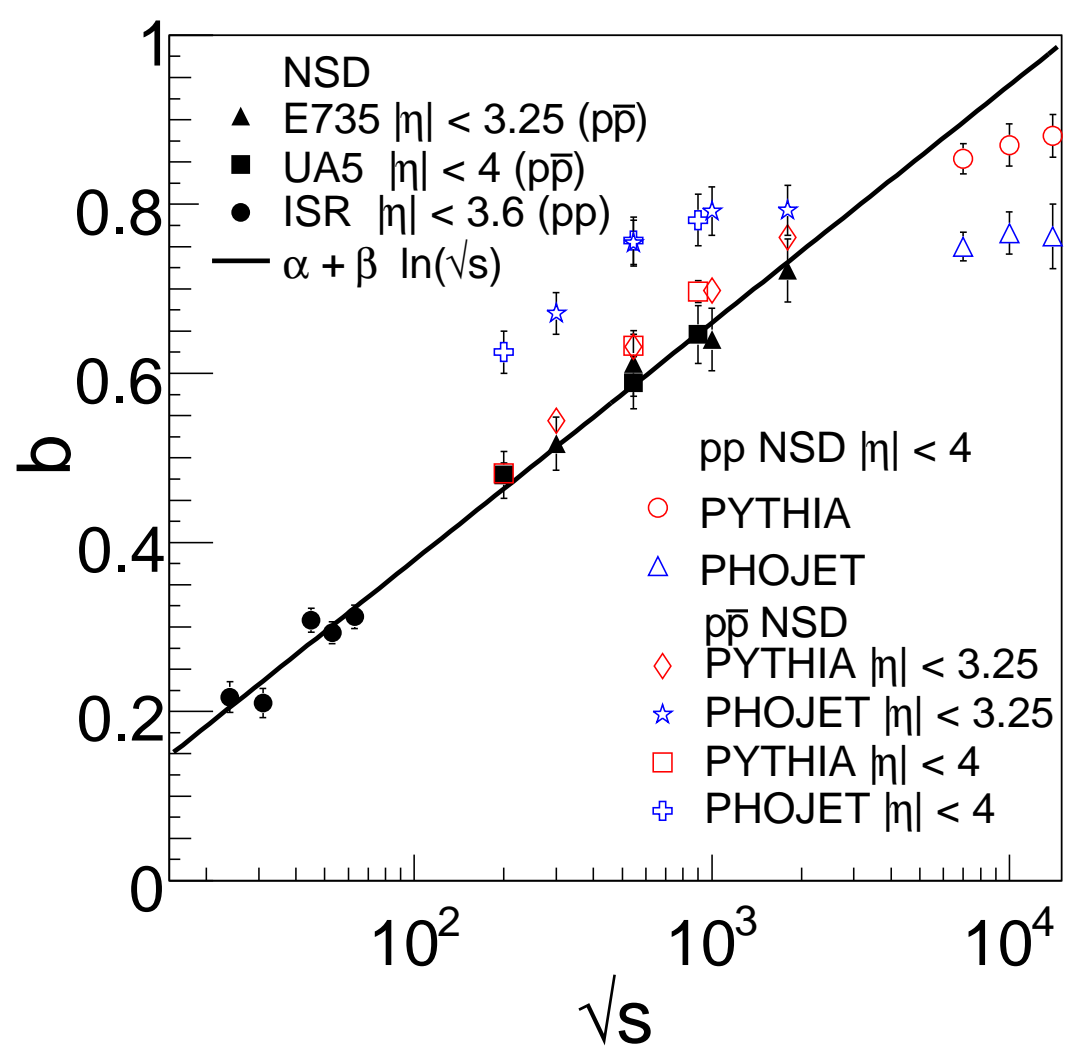

Fig. 7. Correlation strength, $b$ as a function of $\ln \sqrt{s}$ for experimental data from ISR, UA5 and E735 Collaborations. Also shown are comparisons from PYTHIA and PHOJET model calculations, including those expected at $\sqrt{s}=7,10$ and $14 \mathrm{TeV}$. Solid line is a fit to the experimental data points, extrapolation of which indicates the correlation strength will reach a maximum value of unity beyond LHC energies.

components: soft and semi-hard in $p+p(\bar{p})$ collisions 20 . This picture at higher energies is supported by the need of two negative binomial distributions (with different parameters) to explain the multiplicity distribution of produced charged hadrons 30 . Further in such an approach the particle production is thought to be from a certain number of independent initial sources decaying to final products. The produced particles from each source are expected to all stay in the same hemisphere. However certain leakage of particles to the other hemisphere is allowed and is controlled by a leakage parameter in the model. Such a model predicts a saturation of correlation co-efficient at LHC energies 20. The final value of $b$ depends on whether the leakage parameter increases with $\sqrt{s}$ (higher $b$ ), constant with $\sqrt{s}$ or decreases with $\sqrt{s}$ (lower $b$ ). The prediction from this model agrees well with the results from PHOJET 


\begin{tabular}{ccc}
\hline Data/Model & $\alpha^{\prime}$ & $\beta^{\prime}$ \\
\hline Data (UA5, E735, ISR) & $-0.11 \pm 0.12$ & $0.39 \pm 0.02$ \\
Lim & $0.12 \pm 0.07$ & $0.33 \pm 0.02$ \\
PYTHIA & $-0.93 \pm 0.08$ & $0.49 \pm 0.01$ \\
PHOJET & $-3.7 \pm 0.2$ & $1.01 \pm 0.02$ \\
\hline
\end{tabular}

model and is lower than PYTHIA expectations.

\section{Cluster Production}

One way to interpret the forward-backward correlations is through the correlations between particles originating from clusters. The average number of particles originating from clusters is called the cluster size and can be obtained by two methods.

- Method 1: By defining an asymmetry parameter, $Z=N_{f}-N_{b}$, then $\left\langle Z^{2}\right\rangle=r N_{c h}$. Where $r$ is the cluster size and $N_{c h}$ is total number of charged particles.

- Method 2: Directly from the measurement of the correlation coefficient as, $b=\frac{\left\langle N_{c h}\right\rangle / k+1-r}{\left\langle N_{c h}\right\rangle / k+1+r}$, where $\left\langle N_{c h}\right\rangle$ and $k$ are the parameters of a negative binomial fit to the multiplicity distributions 30 .

Figure 8 shows the typical relation between $\left\langle Z^{2}\right\rangle$ and $N_{c h}$ for $p+\bar{p}$ collisions at $\sqrt{s}=900 \mathrm{GeV}$ from PYTHIA. The distribution is fitted by a linear function (dashed and solid lines) in a fixed interval of $N_{c h}$ to extract the cluster size, $r$. We followed this procedure to obtain cluster size from both the models PYTHIA and PHOJET for various $\sqrt{s}$.

Figure 9 shows the $\sqrt{s}$ dependence of the cluster size from E735 $\frac{26}{}$, UA5 27 and ISR 29. The results have been compared to model calculations from PYTHIA and PHOJET, as well as from a theoretical calculations by Lim et al 31. The PYTHIA gives similar cluster size as seen by experiments upto $1.8 \mathrm{TeV}$ and PHOJET give similar cluster sizes as seen by experiments up to $\sqrt{s}=546 \mathrm{GeV}$. Beyond this energy cluster size from PHOJET is higher. The cluster size dependence on $\sqrt{s}$ for the measured experimental data can be parameterized as $\alpha^{\prime}+\beta^{\prime} \ln \sqrt{s}$. The values $\alpha^{\prime}$ and $\beta^{\prime}$ for the experimental data, PYTHIA, PHOJET and from model by Lim et al., are given in the Table. The expectations for top LHC energies from the two models are also shown. PHOJET expects the average number of particles from a cluster to be around 5-6, while PYTHIA gives a much lower value of around 3.1. Thereby providing a clear observable, when compared to LHC data, will distinguish between the underlying mechanism of particle production in $p+p$ collisions.

\section{Summary}

We have reviewed the existing data on forward-backward correlations in $p+\bar{p}(p)$ collisions. Compared these experimental measurements to model calculations from 


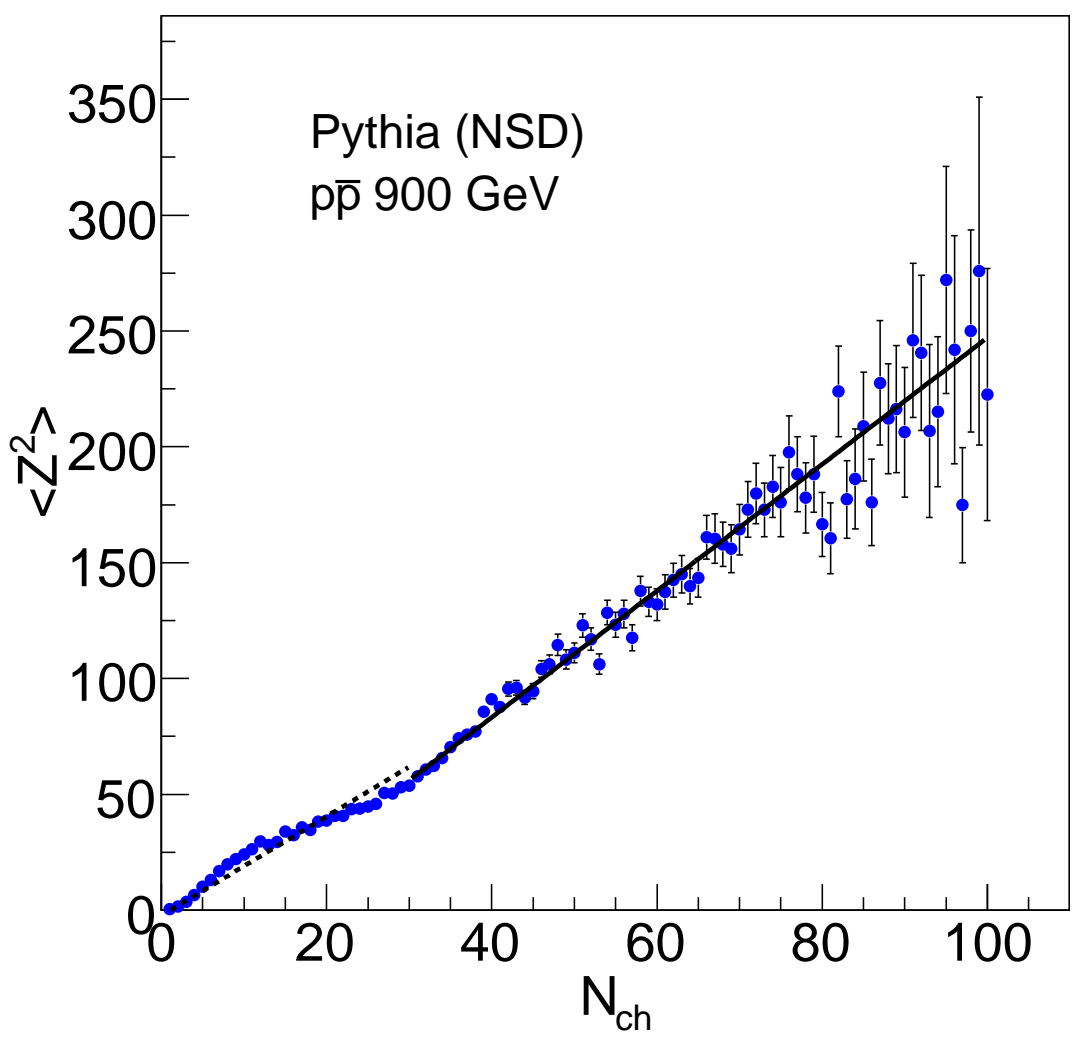

Fig. 8. Variance of the asymmetry measurement $\left(\left\langle Z^{2}\right\rangle\right)$ as a function of charged particle multiplicity for $p+\bar{p}$ collisions at $\sqrt{s}=900 \mathrm{GeV}$ from PYHTIA model. The solid and dashed lines are a linear fit to extract the cluster sizes in various $N_{c h}$ ranges.

PYTHIA and PHOJET. It is found that the correlation strength from PYTHIA is in agreement with the existing measurements, while those from PHOJET give higher correlations. However for top LHC energies of $\sqrt{s}=7,10,14 \mathrm{TeV}$, the correlation strength from PHOJET is lower compared to PYTHIA, suggesting a transition at an intermediate energy accessible at LHC. The measured correlation strength are found to increase linearly with $\ln \sqrt{s}$ and extrapolation suggests it will reach unity (maximum value) around $\sqrt{s}=20 \mathrm{TeV}$, beyond the beam energy reach at LHC. However model calculations suggest the correlation values tends to saturate starting at $\sqrt{s}=2 \mathrm{TeV}$. If such a saturation is observed at LHC it could mean interesting physical consequences related to clan structures in particle production. We have also reviewed the existing results on a common interpretation of forwardbackward correlations in terms of cluster production. The cluster sizes are found to 


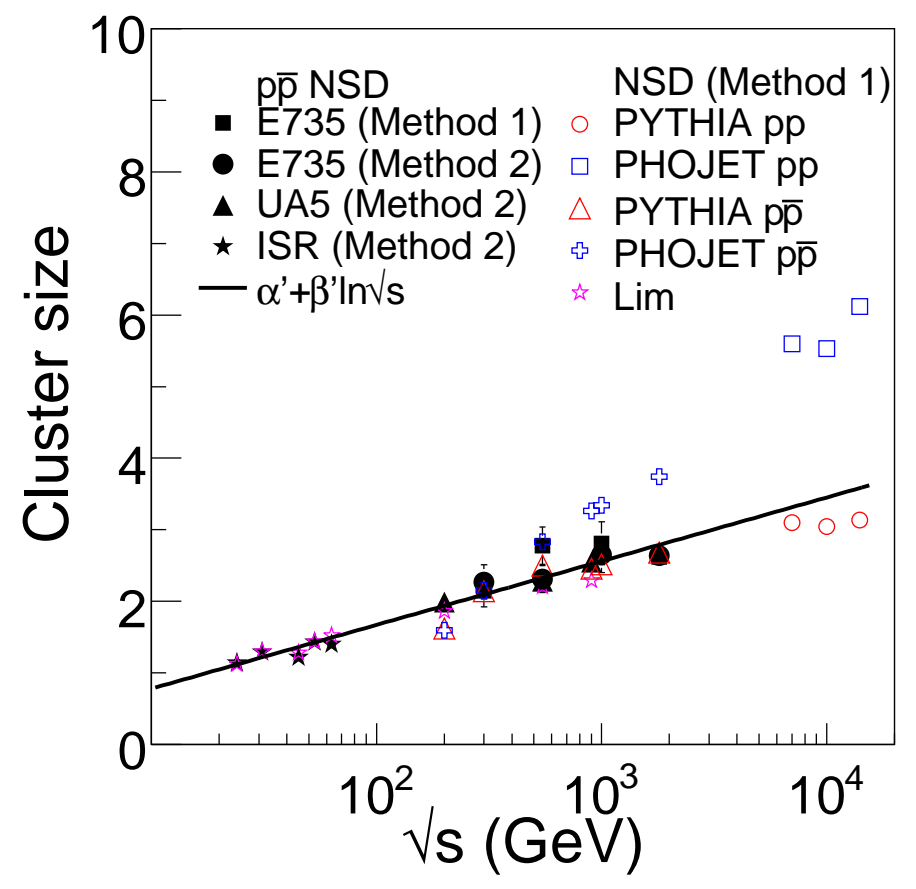

Fig. 9. Cluster size as a function of $\sqrt{s}$ as measured in E725, UA5 and ISR experiments. The method 1 and 2 are discussed in the text. Also shown are the model comparisons with the data at various beam energies in $\mathrm{p}+\mathrm{p}$ collisions and the expectations at top LHC energies from PYTHIA and PHOJET.

increase with increase in beam energy. Similar to the correlation strength, the cluster size from PYTHIA compares well with the existing experimental data. For higher energies (> $546 \mathrm{GeV}$ ) PHOJET gives a higher cluster size compared to PYTHIA. The study of cluster size from forward-backward correlations can be a very good discriminator for the particle production models in $p+p$ collisions.

\section{Acknowledgments}

This work is supported by the DAE-BRNS project sanction No. 2010/21/15BRNS/2026.

\section{References}

1. R. Loren and D. B. Benson, J. Comput. System Sci. 27, 400 (1983).

2. K. Aamodt, et al., ALICE Collaboration, Eur. Phys. J. C 65 (2010) 111.

3. K. Aamodt, et al., ALICE Collaboration, Eur. Phys. J. C 68 (2010) 89.

4. K. Aamodt, et al., ALICE Collaboration, Eur. Phys. J. C 68 (2010) 345.

5. V. Khachatryan, et al., CMS Collaboration, JHEP 1002 (2010) 041. 
6. V. Khachatryan, et al., CMS Collaboration, Phys. Rev. Lett. 105 (2010) 022002.

7. G. Aad, et al., ATLAS Collaboration, Phys. Lett. B 688 (2010) 21.

8. T. Sjostrand, et al., Computer Physics Commun. 135 (2001) 238.

9. T. Sjostrand and M. van Zijl, Phys. Rev. D 36 (1987) 2019.

10. T. Sjostrand and P. Skands, Eur. Phys. J. C 39 (2005) 129.

11. T. Sjostrand and P. Skands, JHEP 06 (2006) 026.

12. R. Engel, Z. Phys. C 66 (1995) 203.

13. R. Engel, J. Ranft and S. Roesler, Phys. Rev. D 52 (1995) 1459.

14. A. Capella, et al., Phys. Rep. 236 (1994) 225.

15. A. Capella and A. Krzywicki, Phys. Rev. D 18 (1978) 4120.

16. P. Carruthers and C.C. Shih, Phys. Lett. B 165 (1985) 209:

17. M. A. Braun, C. Pajares and V.V. Vechernin, Phys. Lett. B 493 (2000) 54.

18. A. Capella and J. Tran Thanh Van, Z. Phys. C 18 (1983) 85.

19. J. Dias de Deus, J. Kwiecinski and M. Pimenta, Phys. Lett. B 202 (1988) 397.

20. A. Giovannini and R. Ugoccioni, Phys. Rev. D 60 (1999) 074027; Phys. Rev. D 59 (1999) 094020.

21. R. C. Hwa and C. B. Yang, arXiv:0705.3073

22. B. I. Abelev, et al., STAR Collaboration, Phys. Rev. Lett. 103 (2009) 172301.

23. Y. V. Kovchegov, E. Levin and L. McLerran, Phys. Rev. C 63 (2001) 024903.

24. N. Armesto, L. McLerran and C. Pajares, Nucl. Phys. A 781 (2007) 201.

25. P. Brogueira, J. Dias de Deus and C. Pajares, Phys. Lett. B 675 (2009) 308.

26. T. Alexopoulos, et al., E735 Collaboration, Phys. Lett. B 353 (1995) 155.

27. R. E. Ansorge, et al., UA5 Collaboration, Z. phys. C 37 (1988) 191.

28. B.K. Srivastava, STAR Collaboration, Int. J. Mod. Phys. E 16 (2008) 3371.

29. S. Uhlig, et al., Nucl. Phys. B 132 (1978) 15.

30. A. K. Dash and B. Mohanty, J. Phys. G 37 (2010) 025102.

31. S. L. Lim et al., Z. Phys. C 43 (1989) 621. 